\title{
Grundkurs Phytotherapie
}

\section{Termin: 5.-7. Juni 2008}

Ort: $\quad$ Engelberg

Anmeldung: bis zum 15. April 2008

(mind. 20 Teilnehmer erforderlich)

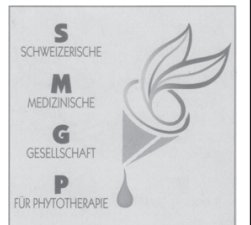

\section{Themen:}

- Herstellung von Phytopharmaka

- Qualitätsaspekte

- Phytotherapeutisches Grundsortiment

- Literatur

- Phytotherapie im schweizerischen Gesundheitswesen

(insbesondere Regelungen des Heilmittelgesetzes und bei den Sozialversicherungen)

\section{Ziele:}

- Vertiefte Auseinandersetzung mit Phytotherapie

- Erlangen von Basiswissen über Arzneipflanzen

- Kennenlernen der Arzneipflanzen in ihrer natürlichen Umgebung

\section{Anmeldung und weitere Informationen}

Zürcher Hochschule für Angewandte Wissenschaften Frau Irène Tinguely

Postfach, $\mathrm{CH}-8820$ Wädenswil

Telefon +41 (0)5893459 80, Fax +41 (0)589345001

E-Mail: kurse-smgp.Isfm@zhaw.ch www.smgp.ch

\section{KARGER}

\section{Hier könnte Ihr Fort- und Weiterbildungsangebot stehen!}

Nutzen Sie FORSCHENDE KOMPLEMENTÄRMEDIZIN zur effektiven Kommunikation mit Ihrer Zielgruppe! So schaffen Sie die besten Voraussetzungen sowohl gedruckt als auch online gesucht und gefunden zu werden.

Informationen und Angebote bei: Verena Hering

Tel. 0761/45207-19

v.hering@karger.de 